\title{
Top-down attentional processing enhances auditory evoked gamma band activity
}

\author{
Stefan Debener, ${ }^{\text {1,2,CA }}$ Christoph S. Herrmann, ${ }^{3,4}$ Cornelia Kranczioch,, Daniel Gembris' and \\ Andreas K. Engel ${ }^{1,2}$
}

\begin{abstract}
'Cellular Neurobiology Group, Institute of Medicine, Research Center Juelich, 52425 Juelich; ${ }^{2}$ Institute of Neurophysiology, University Hospital, Eppendorf, 20246 Hamburg; ${ }^{3}$ Max-Planck-Institute of Cognitive Neuroscience, 04303 Leipzig; ${ }^{4}$ Department of Biopsychology, Institute of Psychology, University of Magdeburg, 39016 Germany

${ }^{\mathrm{CA} C}$ Corresponding Author: s.debener@fz-rjuelich.de
\end{abstract}

Received 20 September 2002; accepted 6 February 2003

DOI: 10.1097/0I.wnr.0000064987.96259.5c

\begin{abstract}
In contrast to animal studies, relatively little is known about the functional significance of the early evoked gamma band activity in humans. We investigated whether evoked and induced $40 \mathrm{~Hz}$ activity differentiate automatic, bottom-up aspects of attention from voluntary, top-down related attentional demands. An auditory novelty-oddball task was applied to 14 healthy subjects. As predicted, more evoked gamma was found for the target condition than in the two task-irrelevant conditions. Since gamma band activity was not
\end{abstract}

enhanced for novel stimuli, the evoked gamma response cannot be explained with a simple concept of stimulus arousal. Neither induced gamma nor the degree of $40 \mathrm{~Hz}$ phase-locking were different between the experimental conditions. Taken together, our data emphasize the role of evoked gamma band activity for top-down attentional processing. NeuroReport 14:683-686 (c) 2003 Lippincott Williams \& Wilkins.

Key words: Attention; Auditory system; Bottom-up; Gamma band response; Top-down

\section{INTRODUCTION}

Accumulating evidence suggests that gamma oscillations in the $30-80 \mathrm{~Hz}$ frequency range serve as a mechanism for binding various features of an object to a unitary whole $[1,2]$. Pioneering work in this field has focused on analyzing synchronized high-frequency oscillations in intracranial recordings of few neurons from the cortex of various species $[3,4]$, and more recent research substantiated the claim that similar phenomena can also be recorded non-invasively from the human scalp [5]. Interestingly, human EEG studies unveiled that the magnitude of the early gamma response, which has often been reported to center around $40 \mathrm{~Hz}$, is modulated by attention. For instance, visual [6,7] and auditory target stimuli [8,9] evoke a significantly larger gamma response than do visual and auditory non-target stimuli, respectively. Although not always observed [10,11], the overall pattern of results suggests that selective attention plays a major role in the functional modulation of highfrequency oscillations $[2,12,13]$. This, in turn, is in good agreement with the hypothesis that selective attention helps to solve the binding problem [14,15]. In this regard, selective attention may be understood as part of a rich set of topdown processing mechanisms that subserve integrative functions [2].

Top-down controlled attentional selection should be distinguished from automatic, stimulus-related attention
[16]. In contrast to target processing, which demands topdown attentional selection, the response to novel stimuli can be characterized as bottom-up information processing that is primarily controlled by the sensory input. It is not yet known whether this distinction between top-down and bottom-up influences is reflected in the early gamma band response. The present study analyzed target versus novelty processing with regard to the evoked gamma band response. To this end, a novelty oddball paradigm [17], in which rare task-irrelevant environmental sounds are interspersed in an active oddball task, was repeatedly applied. Based on theoretical considerations [2] and on empirical findings [6-8], we predicted a larger evoked gamma band response for attended targets than for unattended, i.e. taskirrelevant, frequent stimuli. This pattern was hypothesized to be specific for top-down processing and, therefore, enhanced gamma was not expected to occur for the taskirrelevant novel stimuli.

\section{MATERIALS AND METHODS}

After informed consent had been obtained, a total of 14 healthy subjects (mean age $30.14 \pm 4.04$, nine males, 12 right-handed), free of past or current psychiatric or neurological conditions and with normal hearing participated. EEG was recorded using a high impedance 64 
channel system (Electrical Geodesics, Inc., Eugene, Oregon) with a geodesic sensor net (GSN), and $\mathrm{Cz}$ as reference. Subjects sat in a dimly lit, sound-attenuated and electrically shielded chamber. Sensor impedances were $<30 \mathrm{k} \Omega$ prior to data acquisition, band pass $0.1-100 \mathrm{~Hz}$, and the digitized signal $(500 \mathrm{~Hz}$ sampling rate, $0.024 \mu \mathrm{V}$ precision) stored for off-line analysis.

Auditory stimuli were presented binaurally at about $70 \mathrm{~dB}(\mathrm{SPL})$ by using foam-protected air-tube earphones. Two sine tones of 350 and $650 \mathrm{~Hz}(340 \mathrm{~ms}$ duration, $10 \mathrm{~ms}$ rise time, $30 \mathrm{~ms}$ fall time) served as frequent and target tones (counterbalanced across subjects), respectively. The subject's task was to silently count the target tones, which were presented in a pseudo-randomized order. Target probability was $p=0.10$, and frequent probability was $p=0.80$. In addition, 72 unique environmental sounds [18] were presented, comprising a class of novel stimuli $(p=0.10)$. Four blocks with 180 stimuli each were presented (stimulus onset asynchrony 1300-1700 ms, 1 min breaks between blocks). A retest session was scheduled 7 days later at the same time of day. Apart from block order, which was reversed, the same protocol was applied at the second session.

EEG data analysis started with linear interpolation of bad channels $(0.8 \%$ of all channels had to be replaced). Data were $0.5 \mathrm{~Hz}$ high-pass filtered and rejected as artifactual whenever the s.d. within a $200 \mathrm{~ms}$ interval exceeded $20 \mu \mathrm{V}$ on any of the channels. Artifact-free trials (confirmed by visual inspection) were re-referenced to the common average of all sensors and analyzed separately for the first and second half of each condition and for each session.

Wavelet analysis was used in order to provide a continuous measure of the amplitude of a frequency component [19]. The method applied here has been described elsewhere in full detail [6]. Statistical analysis was focused on gamma band activity using a $40 \mathrm{~Hz}$ centered Morlet wavelet. To reveal the evoked fraction of gamma activity which is, by definition, strictly phase-locked to the stimulus, the wavelet transform was performed on the averaged evoked potential waveform. In addition, the wavelet transform was performed for each individual trial, and the resulting values were averaged. This measure reflects the total activity for a certain frequency range, irrespective of whether it is phase-locked to the stimulus or not. Induced activity, which is commonly regarded as representing non-phase-locked activity [20], could then be derived by subtracting the evoked activity from the total measure. Since the degree of gamma phase-locking may vary with task conditions [9], phase-locking factors were computed as follows. The phase of the complex $40 \mathrm{~Hz}$ wavelet decomposition in each single trial was represented as a point on the unit circle irrespective of its amplitude. Averaging these points yields a value of 1 for strictly phaselocked trials and zero for randomly distributed phases [21].

Data were epoched from $-200 \mathrm{~ms}$ before to $800 \mathrm{~ms}$ after stimulus onset. The mean of the -200 to $-50 \mathrm{~ms}$ baseline interval was subtracted from each average [6], and evoked gamma activity was quantified as the maximum peak between 20 and $80 \mathrm{~ms}$. This time window was chosen on the basis of previous knowledge about the time course of the auditory evoked gamma response [8]. The induced gamma response follows the evoked response [9,11], yet, less information about the precise time course of this signal is available. Hence, a sliding time window approach was chosen, where the means of 10 consecutive $50 \mathrm{~ms}$ time intervals between 100 and $600 \mathrm{~ms}$ were analyzed separately. To account for type I error inflation due to multiple testing, the Bonferoni-Holm procedure was applied.

Since a relatively low signal-to-noise ratio (SNR) was evident at many sensors, a full topographical analysis was not performed. The mean SNR, quantified as the evoked signal divided by the standard deviation of the pre-stimulus interval, was 1.75 at lateral sensor sites. In order to avoid a loss of statistical power, and in agreement with previous work, statistical analysis was narrowed on the fronto-central scalp region, where the early evoked gamma response can be identified easily [8-11]. Three fronto-central adjacent sensors $(\mathrm{E} 05, \mathrm{E} 55, \mathrm{Cz})$ had a mean SNR of 2.41, and were therefore chosen to define a central region of interest (ROI). Evoked gamma, induced gamma, and gamma phase-locking activity were submitted separately to three-way ANOVAs with the repeated-measurement factors block (first vs second experimental half), session (first vs second recording session), and condition (frequent, target, and novel condition). The former two factors explored whether the predicted condition effects were temporally robust within (block factor) and across (session factor) experimental sessions. In order to control whether time-locked lowfrequency activity in general, or harmonics of EEG alpha activity in particular, were responsible for the observed $40 \mathrm{~Hz}$ gamma effects [22], two control ANOVAs were calculated. One ANOVA was performed for a $10 \mathrm{~Hz}$ wavelet, and a second analysis was computed for the P1 eventrelated potential (ERP) component (maximum positive peak between 20 and $80 \mathrm{~ms}$ ). ERPs were $25 \mathrm{~Hz}$ low-pass filtered prior statistical analysis. Greenhouse-Geisser (GG) corrected $p$ values are reported where appropriate.

\section{RESULTS}

The time course of the $40 \mathrm{~Hz}$ gamma band responses and their phase-locking are presented together with the ERPs in Fig. 1. As can be seen in the left column, all three conditions evoked a small P1 (peak latency $48 \mathrm{~ms}$ ) followed by a prominent N1 ERP (106 ms), which was slightly larger for target than for novel and frequent stimuli. Novel stimuli evoked a novelty-P3 component, whereas target stimuli evoked a P3b (for a detailed discussion of the ERP results see [23])

Wavelet analysis revealed a prominent evoked gamma band response which raised shortly after stimulus onset and peaked at about $60 \mathrm{~ms}$. In agreement with previous studies [8-10], this response was largest at fronto-centrally located sensors. As indicated by a significant condition main effect $(\mathrm{F}(2,26)=4.64, p<0.05$; GG $\epsilon=0.60)$, frequent, target and novel conditions differed with regard to the amount of gamma band activity. Session and block main effects were not significant $(F<1)$, and only the session $\times$ block interaction approached a marginal significance level $(F(1,13)=3.17, p=0.10)$ but this was not analyzed further. The significant condition main effect is illustrated in Fig. 2. Planned comparisons confirmed significantly more evoked gamma for targets as compared to frequents $(\mathrm{t}(13)=2.55$, 


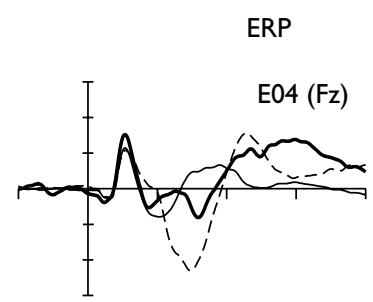

Evoked $40 \mathrm{~Hz}$

Induced $40 \mathrm{~Hz}$

$40 \mathrm{~Hz}$ Phase-locking
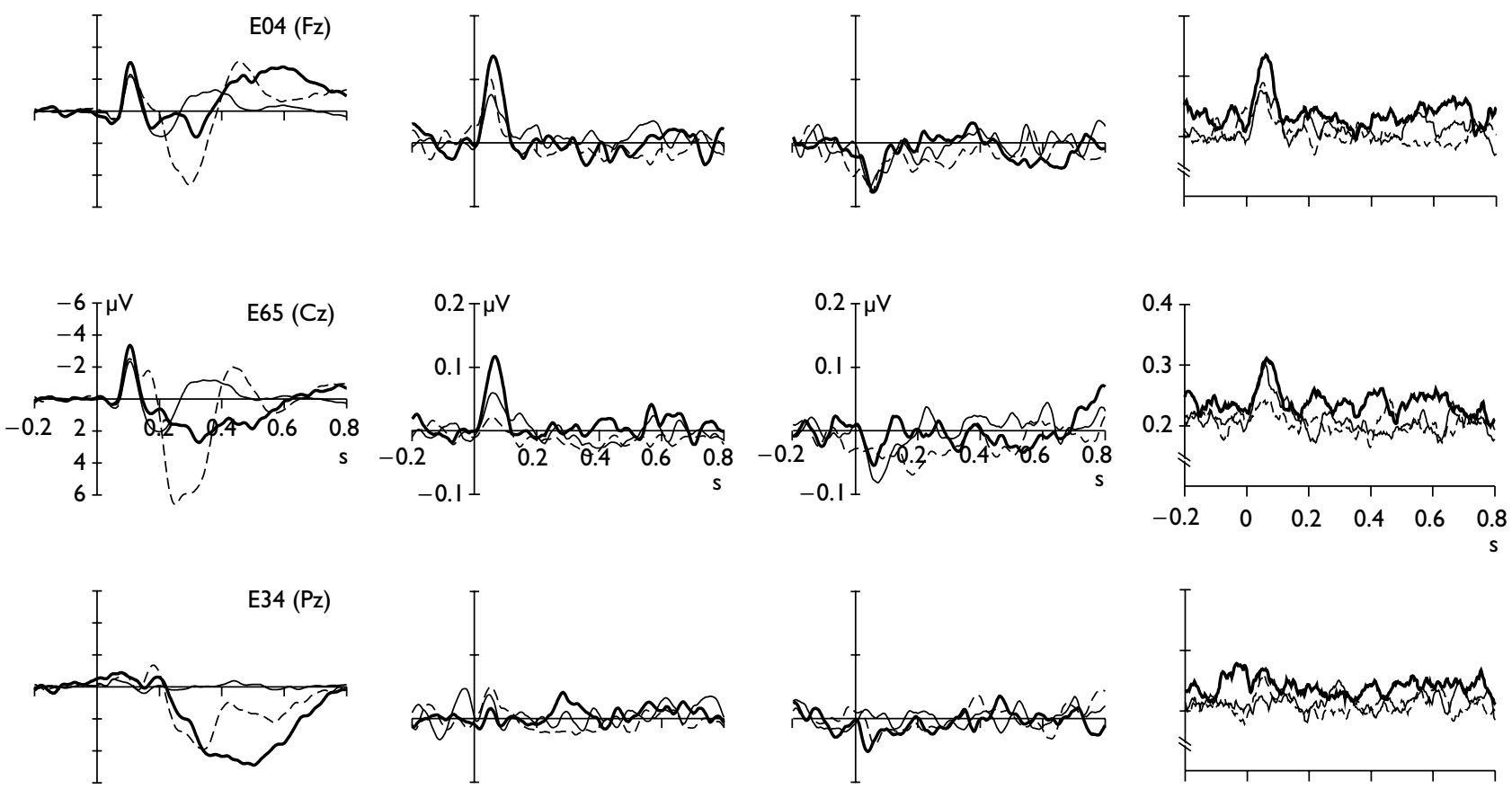

- target frequent $\quad-----\cdot$ novel

Fig. I. Event-related potentials (ERPs, left column), evoked $40 \mathrm{~Hz}$ activity (second column), induced $40 \mathrm{~Hz}$ activity (third column), and $40 \mathrm{~Hz}$ phaselocking (right column) are shown for three midline sensors (corresponding 10 - 10 scalp sites in brackets). Data shown are pooled across both blocks and both recording sessions. Conditions were rare target tones (thick line), task-irrelevant frequent tones (thin line), and task-irrelevant novel environmental sounds (dashed line). Note the different scaling and polarity for the different measures. Absolute phase-locking values, and baseline-corrected ERPs and $40 \mathrm{~Hz}$ activities are shown.

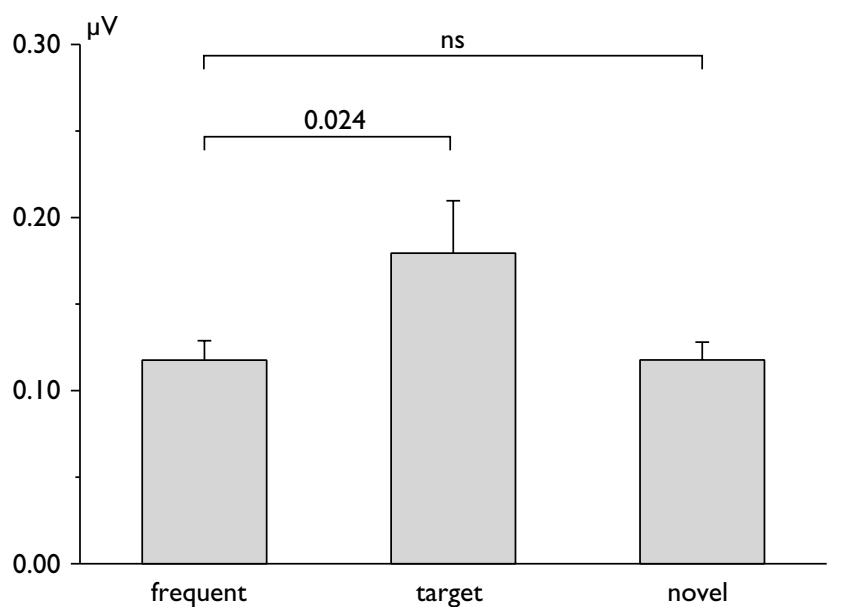

Fig. 2. Mean ( \pm s.e.m.) auditory evoked gamma band activity for target, and task-irrelevant frequent and novel stimuli, as revealed by a $40 \mathrm{~Hz}$ Morlet wavelet analysis. Data shown for the fronto-central midline $\mathrm{ROI}$ are scaled to $\mu \mathrm{V}$ (for details, see [6]), and are aggregated across both blocks and both recording sessions.

$p<0.05)$. Novel stimuli, on the other hand, did not differ from frequents with respect to the amount of evoked gamma activity $(\mathrm{t}(13)<1$, ns).
The induced gamma band response is also shown in Fig. 1 (third column). The decrease of induced gamma shortly after stimulation onset reflects the subtraction of the evoked fraction from the overall gamma band activity. Importantly, a genuine induced gamma band response was not obtained. Only one of the 10 induced gamma band analyses revealed a condition main effect (150-200 ms time window, $\mathrm{F}(2,26)=4.32, p<0.05)$, but this effect did not survive type I error correction. None of the later time intervals disclosed any further condition effects.

The time course of EEG gamma phase-locking is shown in the right column of Fig. 1. An enhanced early peak at frontocentral sensors was found for all three experimental conditions. Presumably, this peak reflects the amount of $40 \mathrm{~Hz}$ phase-locking contributing to the evoked response. Regarding the degree of phase-locking, statistically significant differences between target, frequent and novel conditions could not be observed. Accordingly, the corresponding ANOVA on the baseline corrected phase-locking peak did not reveal any significant main or interaction effect (all $\mathrm{F}<1$ )

Post-hoc analysis explored whether the present results could be limited to the gamma band frequency range. A block $\times$ session $\times$ condition ANOVA was performed for the signal convolved with a $10 \mathrm{~Hz}$ wavelet. This analysis did not reveal a significant condition main effect, or any other 
statistically relevant effect (all $p>0.10$ ). In order to further rule out that the time domain ERPs reflected the same effects as the gamma analysis, P1 amplitudes were analyzed (see Fig. 1). Yet again, the condition $\times$ session $\times$ block ANOVA for the P1 peak did not reveal any significant main or interaction effect (all $p>0.10$ ).

\section{DISCUSSION}

The present study has investigated EEG gamma activity in an auditory novelty oddball task. In addition to rare target and frequent standard tones (conventional oddball), taskirrelevant unique environmental sounds were presented in this paradigm, and therefore, the novelty oddball allows the investigation of both goal-directed (target processing) and stimulus-driven (novelty processing) attention. As predicted, a significantly enhanced evoked gamma band activity was observed in response to rare targets. In contrast, novel stimuli did not cause an enhancement of the gamma response. This observation confirms and extends previous reports [8] and supports the view of EEG gamma as a correlate of higher-order cognitive processes such as attention. In addition to previous evidence, our results suggest that the enhanced evoked gamma response is rather specifically related to top-down attentional processing. Accordingly, this result is in line with theoretical considerations of the evoked gamma response as reflecting a matching process between the template stimulus held in working memory and the incoming, just perceived stimulus $[2,7]$.

In contrast to previous auditory oddball $40 \mathrm{~Hz}$ analyses, we did not find convincing evidence for a genuine induced gamma band response. Only in the time interval 150-200 ms post-stimulus onset was a trend revealed towards a reduced induced activity for novel environmental sounds. Similar effects have not yet been reported. Even on a descriptive level, previous studies reporting a late-induced gamma band activity $[10,11]$ could not be replicated. However, note that these previous reports are inconsistent with regard to the direction of the target-induced gamma response.

The degree of phase-locking provides additional information not fully covered by the analysis of evoked and induced auditory gamma activity $[6,8,9]$. In line with other studies [11], stronger phase-locking during the time course of the early gamma response was observed. Even though merely phase-locked activity builds up the evoked measure, phase-locking could not be held responsible for the condition effects found in evoked gamma activity. In other words, the enhanced evoked gamma response for target stimuli was not due to a diminished latency jitter between target trials. Rather, partly different neural networks involved in condition-specific task processing should be held responsible for this effect.

\section{CONCLUSION}

The present report replicates previous findings on an enhanced early evoked gamma band activity for target as compared to non-target auditory stimuli. Moreover, novel stimuli, which are known to involuntarily capture attention also did not enhance the early evoked gamma band response. Contrary to what has been discussed previously, a different amount of phase-locking was not responsible for this pattern of results. In conclusion, our findings provide further evidence that the early evoked gamma band activity does not just reflect stimulus-driven bottom-up processes, but also top-down related aspects of attention. Hence, the question emerges to what extent the observed activity originates from the partly segregated anatomical networks associated with top-down and bottom-up attention [16]. Unfortunately, the topographical information provided by $40 \mathrm{~Hz}$ oscillations in the human EEG is limited, and still a matter of dispute [24]. Therefore, future work in this field will benefit from combining fMRI and $40 \mathrm{~Hz}$ EEG recordings.

\section{REFERENCES}

1. Singer W and Gray CM. Annu Rev Neurosci 18, 555-586 (1995).

2. Engel AK, Fries P and Singer W. Nature Rev Neurosci 2, 704-716 (2001).

3. Gray CM, König P, Engel AK and Singer W. Nature 338, 334-337 (1989).

4. Kreiter AK and Singer W. J Neurosci 16, 2381-2396 (1996).

5. Mueller MM, Bosch J, Elbert T et al. Exp Brain Res 112, 96-102 (1996).

6. Herrmann CS, Mecklinger A and Pfeifer E. Clin Neurophysiol 110, 636-642 (1999).

7. Herrmann CS and Mecklinger A. Vis Cogn 8, 593-608 (2001).

8. Tiitinen H, Sinkkonen J, Reinikainen K et al. Nature 364, 59-60 (1993).

9. Yordanova J, Kolev V and Demiralp T. Neuroreport 8, 3999-4004 (1997).

10. Fell J, Hinrichs H and Roeschke J. Neurosci Lett 235, 121-124 (1997).

11. Gurtubay IG, Alegre M, Labarga A et al. Clin Neurophysiol 112, 1219-1228 (2001).

12. Herrmann CS and Knight RT. Neurosci Biobehav Rev 25, 465-476 (2001).

13. Fries P, Reynolds JH, Rorie AE and Desimone R. Science 291, 1560-1563 (2001).

14. Treisman A. Curr Opin Neurobiol 6, 171-178 (1996).

15. Reynolds JH and Desimone R. Neuron 24, 19-29 (1999).

16. Corbetta M and Shulman GL. Nature Rev Neurosci 3, 201-215 (2002).

17. Friedman D, Cycowicz YM and Gaeta H. Neurosci Biobehav Rev 25, 355-373 (2001).

18. Fabiani M and Friedman D. Psychophysiology 32, 579-594 (1995).

19. Samar YJ, Bopardikar A, Rao R and Swartz K. Brain Lang 66, 7-60 (1999).

20. Basar-Eroglu C, Strueber D, Schuermann M et al. Int J Psychophysiol 24, 101-112 (1996).

21. Tallon-Baudry C, Bertrand O, Delpuech C and Pernier J. J Neuroscience 16, 4240-4249 (1996).

22. Juergens E, Roesler F, Hennighausen E and Heil M. Neuroreport 6, 813-816 (1995).

23. Debener S, Kranczioch C, Herrmann CS and Engel AK. Int J Psychophysiol 46, 77-84 (2002).

24. Herrmann CS and Friederici A. Science 292, 163a (2001).

Acknowledgements: We are grateful to $M$. Fabiani for providing us with the environmental sounds, thank $M$. Grigutsch for software support, and O. Haumann for help in data acquisition. 